$12,13,08$

\title{
Вариация состояния поверхности в ходе сканирования в низковольтном РЭМ и ее влияние на размеры рельефной структуры
}

\author{
(С) Ю.В. Ларионов, Ю.В. Озерин
}

Институт общей фризики им. А.М. Прохорова РАН, Москва, Россия

E-mail: luv@kapella.gpi.ru

Поступила в Редакцию 16 октября 2019 г.

В окончательной редакции 21 января 2020 г.

Принята к публикации 21 января 2020 г.

\begin{abstract}
Оценена вариация эмиссии медленных вторичных электронов из поверхности рельефных структур (выступов) в ходе длительного их сканирования в низковольтном растровом электронном микроскопе. Характер вариации зависит от участка профиля выступа, он особенно сложен вблизи углов рельефных структур. В результате искажаются соответствующие участки кривой видеосигнала от выступа, что приводит к увеличению или даже к уменьшению геометрических размеров этих участков. Вариация эмиссии объяснена наведением локальных зарядов в слое естественного окисла на поверхности кремния. Размер участков изменяется также из-за осаждения на поверхности выступа контаминационной пленки. Предположительно, ее осаждение зависит от наведенных зарядов на поверхности выступа и поэтому плохо воспроизводимо. Зафиксирован случай отсутствия контаминационного уширения выступа в результате длительного его сканирования.
\end{abstract}

Ключевые слова: нанометрология, низковольтный растровый электронный микроскоп (РЭМ), рельефная структура, поверхностные зарядовые состояния, контаминация.

DOI: 10.21883/FTT.2020.06.49356.608

\section{1. Введение}

Низковольтные растровые электронные микроскопы (РЭМ) используются для контроля критических размеров при производстве интегральных микро и наносхем. Однако, при контроле элементов с нанометровыми размерами с помощью низковольтных РЭМ возникают неожиданные метрологические проблемы [1]. Например, в [2] обнаружено, что в ходе длительного сканирования РЭМ-значение ширины верхнего основания (BО) выступов может даже уменьшаться. Вариация размера в ходе сканирования рельефной структуры требует теоретического объяснения и создает практические сложности в оценке действительного значения ширины.

Вариация значений размеров выступа во времени объяснена в [1] изменением его поверхности. Вопервых, образованием контаминационной пленки на поверхности выступа, увеличивающей его ширину. Вовторых, наведением электрического заряда на участках выступа, способного уменьшать и увеличивать значение ширины.

Образование контаминационной пленки. Оно вызывается осаждением на выступ углеводородных (УВ) частиц. Осаждение УВ-частиц на поверхность вызывается стимулирующим действием падающего электронного пучка. Скорость осаждения зависит от потока частиц в сканируемую область и от тока электронного пучка, т.е. определяется внешними по отношению к поверхности условиями. Но в [1] обнаружилось, что рост пленки зависит также от состояния этой поверхности. В определенных условиях контаминация на поверхности вообще не возникает даже после длительного ее сканирования в РЭМ.

Наведение заряда. Электрический заряд, присутствующий в диэлектрических пленках на сканируемой поверхности, способен изменяться под воздействием падающего электронного пучка [3]. Это особенно значимо на участках рельефной поверхности вблизи углов выступов, где слой пленки обычно толще а плотность зарядов выше, чем на плоской поверхности. В результате там возможно заметное изменение локализованных зарядовых состояний. Из-за их вариации должна изменяться эмиссия медленных вторичных электронов (МВЭ) из соответствующих участков поверхности [3].

Эмиссия МВЭ из сканируемых точек поверхности создает РЭМ-изображение выступа. Из РЭМ-изображения может быть сформирована кривая видеосигнала (BC), которая усредняет изменения эмиссии от деталей профиля вдоль строки развертки по всем или по части строк изображения. На кривой ВС присутствуют пики, соответствующие правой и левой боковым стенкам выступа. РЭМ-значение ширины выступа измеряется по расстоянию между выбранными точками на указанных пиках, которые теоретически (в модели формирования РЭМ-изображения [4]) соответствуют границам верхнего основания (BО) выступа. Из-за наведения зарядов и 
вариации эмиссии МВЭ происходит изменение положения пиков ВС. Поэтому РЭМ-значение ширины выступа должно отличаться от того, которое определяется лишь положением его геометрических границ.

В этом объяснении вариации значений геометрических размеров отсутствуют детали механизма влияния состояния поверхности на РЭМ-изображение выступа и кривые ВС, а также ее влияние на осаждение УВ-частиц на эту поверхность.

Цель настоящей работы - оценить вариацию состояния поверхности однотипных выступов на пластине в результате продолжительных и многократных их сканирований в низковольтном РЭМ. Практически важная составляющая работы - оценить влияние этой вариации поверхности на геометрические размеры выступов.

\section{2. Литературный обзор}

Электрические заряды, способные влиять на эмиссию МВЭ из поверхности, могут локализоваться как в высокоомном кремнии, в котором вытравлены выступы, так и в тонком слое естественного окисла, который вырастает на поверхности кремния при хранении пластины в атмосферных условиях. Наиболее близким модельным объектом для такого образца может быть металл-диэлектрик-полупроводник-структура, в которой отсутствует внешний слой металла $[5,6]$. Толщина диэлектрика в такой структуре простирается от нескольких до нескольких десятков нанометров. Толщина естественного окисла составляет 1.5-2.5 nm [7]. И в таком тонком слое могут располагаться, предположительно, значительная часть зарядовых состояний, которые определяют зарядовые свойства поверхности [8].

Из-за проникновения атомов кислорода в кремниевый образец на его границе возникает переходной слой. На расстоянии $\sim 1-2 \mathrm{~nm}$ от границы раздела находится слой $\mathrm{SiO}$ с нарушенной стехиометрией [8]. За ним с внешней стороны структуры следует стехиометричный слой $\mathrm{SiO}_{2}$ толщиной в несколько нанометров, но с напряженными и непрочными $\mathrm{SiO}-$ связями. При воздействии излучений и под действием механических напряжений в этом слое могут формироваться атомные структуры, насыщенные точечными или сеточными дефектами. Под действием ионизирующей радиации возможно перемещение этих дефектов из слоя окиси кремния к границе кремния [8].

В слое $\mathrm{SiO}$ с нарушенной стехиометрией способны формироваться дефекты с дефицитом кислорода (рядом с границей кремния) и с его избытком во внешнем части этого слоя. К дефектам с дефицитом кислорода (с вакансией мостикового кислорода) относится $E^{\prime}$-центр, представляющий собой атом кремния, связанный только с тремя атомами кислорода и с одним неспаренным электроном. К дефектам с избытком кислорода относится немостиковый кислородно-дырочный центр (NBOHC) и перекисный) и перекисный радикал, в которых между атомами кремния располагаются по 2 атома кислорода. $E^{\prime}$-центры способны захватывать дырки и заряжаться положительно. Центры NBOHC могут заряжаться отрицательно, в частности, при инжекции электронов извне или положительно - при удалении неспаренного электрона. На границе между слоем окисла и кристаллического кремния формируются $P_{B}$-центры. Они представляют собой трехкоординированные атомы кремния с одним неспаренным электроном. Принимая или отдавая электрон они способны заряжать слой положительно или отрицательно [8].

Существенно присутствие водорода в приповерхностных слоях кремния и $\mathrm{SiO}$, приводящее к нейтрализации зарядовой активности поверхностных состояний. (Считается, что при комнатной температуре водород присутствует там в концентрации не менее $10^{17} \mathrm{~cm}^{-3}[8]$.) Однако присутствие водорода и его диффузия в объем приводит к тому, что новое стабильное зарядовое состояние может наступать через сотни $\mathrm{h}$ после радиационного воздействия при $T=300 \mathrm{~K}$.

Наведение зарядов может происходить также в приповерхностной области используемого высокоомного кремния $p$-типа. В ней часть подвижных дырок будет нейтрализована электронами из падающего пучка или вторичными электронами. Глубина проникновения падающих электронов с энергией $0.8 \mathrm{keV}$ (как в низковольтном РЭМе) в кремний составляет до $\sim 20 \mathrm{~nm}$ [9]. Поэтому можно ожидать, что эта приповерхностная часть кремния будет заряжена отрицательно. Часть несвязанных электронов, созданных падающим пучком (в том числе и МВЭ из глубины их выхода из поверхности $\lambda \sim 4 \mathrm{~nm}[10])$ будут эмитированы из поверхности, оставляя после себя наведенный поверхностный заряд. Все эти расположенные слоями и имеющие разный электрический заряд дефекты способны создать плоскую многослойную структуру.

Особенность используемого в экспериментах образца состоит в том, что сканируемая поверхность является не плоской, а рельефной. На боковых стенках выступов также способен образовываться многослойный зарядовый слой, но его параметры должны отличаться от соответствующих слоев горизонтальной поверхности. Критически важным и сложным для теоретического осознания является формирование поверхностных зарядовых слоев на углах верхнего основания выступа. Действительно, изменение эмиссии МВЭ от краев верхнего основания из-за наведенных зарядов должно изменить положение пиков кривой ВС, по положению которых измеряется ширина выступа. Но особенности формирования диэлектрических слоев на углах выступа и эмиссии МВЭ из них пока неизвестны.

Модель формирования зарядовых состояний в объеме окисной пленки и под ней для рельефной поверхности заметно сложнее по сравнению с плоской поверхностью образца $\mathrm{SiO}_{2}$, соответствующая модель для которой представлена в [3]. Общим для этих моделей является 
возможность формирования вдоль поверхности многослойной электрической структуры, которая может существовать до сканирования поверхности электронным пучком, может модифицироваться под его воздействием и сохраняться и даже изменяться во времени после этого воздействия.

На наш взгляд, поверхность выступов, покрытая пленкой естественного окисла, может быть важным фактором, определяющим ее зарядовое состояние. Оно способно быть изменчивым вдоль поверхности выступа и в течении длительного времени из-за плохой воспроизводимости зарядовых состояний в слое окисной пленки, a также из-за изменчивости насыщения ее водородом при перемещении образца в вакуум и обратно. Важно и возможно выявить степень стабильности эмиссии МВЭ из разных участков нанометровых выступов в условиях изменчивости указанных влияющих величин за длительное время наблюдений.

\section{3. Условия эксперимента}

Средством измерения геометрических размеров являлся измерительный РЭМ S9260 (Hitachi) с энергией электронов в пучке $0.8 \mathrm{keV}$ и увеличением $150.000 \mathrm{X}$ (при размере пикселя $-1.75 \mathrm{~nm}$ ). Высокая стабильность и воспроизводимость пучка электронов на этом РЭМе позволила надежно фиксировать изменения эмиссии МВЭ из локальных участков профиля на длительных временных промежутках, что дало возможность оценивать изменения зарядового состояния поверхности. Формирование РЭМ-изображений (сканов) выступов производилось в результате усреднения 32 последовательных кадров их сканирования. Далее на основе усредненного изображения создавалась кривая ВС с дополнительным усреднением ее формы по строкам развертки. И наконец, программа [11] выделяла точки на кривой ВС, которые в модели измерений [4] соответствуют границам участков выступа, в том числе и для участка верхнего основания. По расстоянию между ними измерялись линейные размеры всех участков геометрического профиля выступа. В результате многократного усреднения изображений и строк в нем, „шум“ в значениях ширины верхнего основания составлял всего $0.2-0.3 \mathrm{~nm}$.

Объектами измерения являлись выступы с высотой $\sim 350 \mathrm{~nm}$, полученные с помощью химического травления кремния. Ширина ВО выступа была $\sim 250-290 \mathrm{~nm}$, а углы наклона боковой стенки $\sim 65^{\circ}$. В атмосферных условиях хранения поверхность выступа покрывалась пленкой естественного окисла толщиной $\sim 1-3 \mathrm{~nm}$, на которой в результате ее сканирования в РЭМ должен формироваться слой углеводородной пленки $\mathrm{C}_{n} \mathrm{H}_{n+2}$ (контаминации). Схема профиля выступа [12] представлена на рис. 1.

Поверхностный заряд не мог быть определен непосредственно с помощью использованного РЭМ. Поэтому оценка изменения заряда производились косвенно и

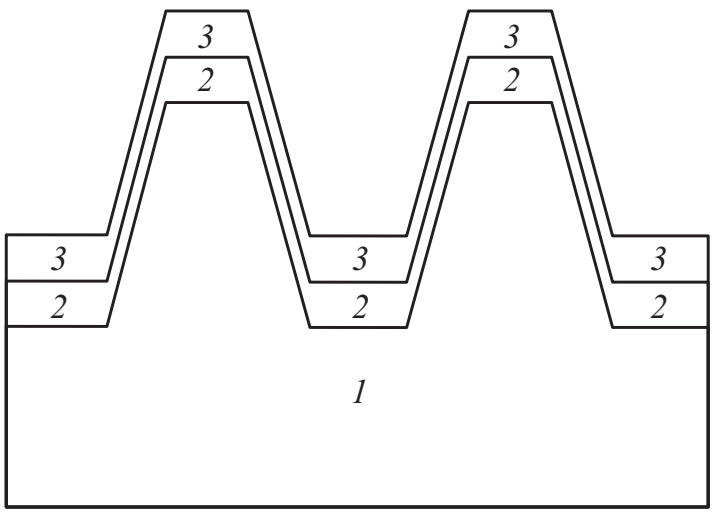

Рис. 1. Схема сечения измеряемого выступа и покрывающих его слоев: $1-\mathrm{Si}, 2-\mathrm{SiO}_{2}-$ слой контаминационной пленки. $3-\mathrm{C}_{n} \mathrm{H}_{n+2}-$ слой контаминационной пленки.

качественно по изменению эмиссии МВЭ, формирующих кривую ВС. Оценка изменения эмиссии МВЭ по кривым ВС позволяла локализовать нанометровые участки профиля, на которых изменяется заряд.

\section{4. Результаты исследования}

В ходе многократных сканирований выступов в течение длительного времени были выявлены закономерности динамики эмиссии МВЭ из разных участков этих выступов. Характер динамики оказался различным для разных выступов на одной пластине, сканированных в одинаковом режиме. На рис. 2 показаны кривые значений ширины $\mathrm{BO}$ двух выступов $(B 1, B 2)$ которые наиболее заметно отличались по своей динамике в ходе сканирования. Кривые формировались по результатам четырех серий сканирования. Каждая серия включала от 50 до 150 сканов и была отделена от соседних серий промежутком времени от нескольких суток до 3-4 месяцев при хранении образца в атмосфере чистой комнаты.

Как видно, начало каждой серии экспонирования совпадает с пиком значений ширины (быстрая фаза изменения размеров), за которым следует их спад для обоих выступов. Характер вариации значений ширины после пиков и до конца сканирований в каждой серии экспонирования на рис. 2, $a$ и $b$ (медленная фаза изменения размеров) резко отличен: в первом случае виден монотонный рост ширины, во втором - слабое уменьшение с дальнейшей стабилизацией значений. Среднее значение ширины выступа $B 2$ в медленной фазе по данным измерений в сериях 4 и 1 уменьшилось на $\sim 0.5 \mathrm{~nm}$. Последний результат свидетельствует о том, что контаминационное уширение выступов может, предположительно, отсутствовать даже после длительного их сканирования (600 сканов или около 20000 кадров) и нескольких выводов пластины из вакуума.

Важные детали динамики эмиссии МВЭ можно извлечь из вариаций формы кривых ВС в ходе последо- 


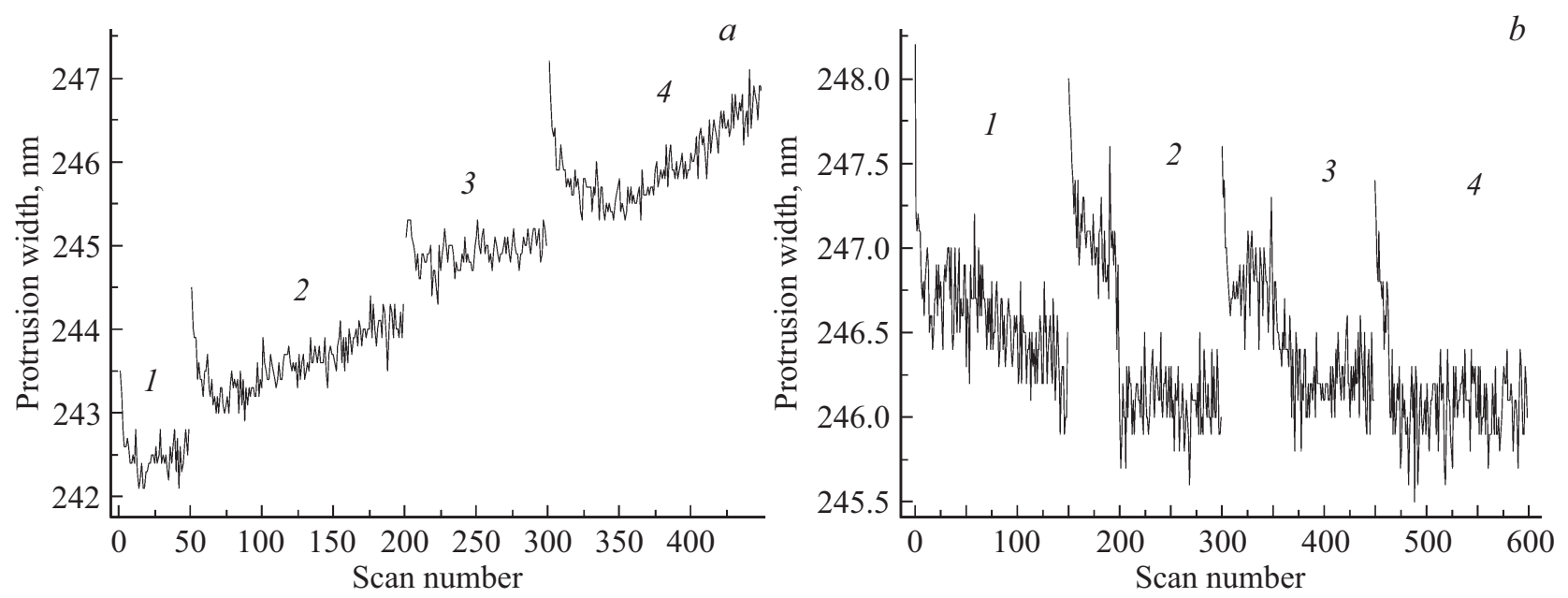

Рис. 2. Зависимости ширины выступов $B 1$ и $B 2$ от номера скана. Серии сканирования $1-4$ разделены промежутками с выводом пластины из РЭМ. Видны пики значений ширины в начале каждой серии. Вне участков ВС с этими пиками заметен монотонный рост значений ширины $(a)$ для $B 1$ или слабый их спад в последовательности серий $(b)$ для $B 2$.
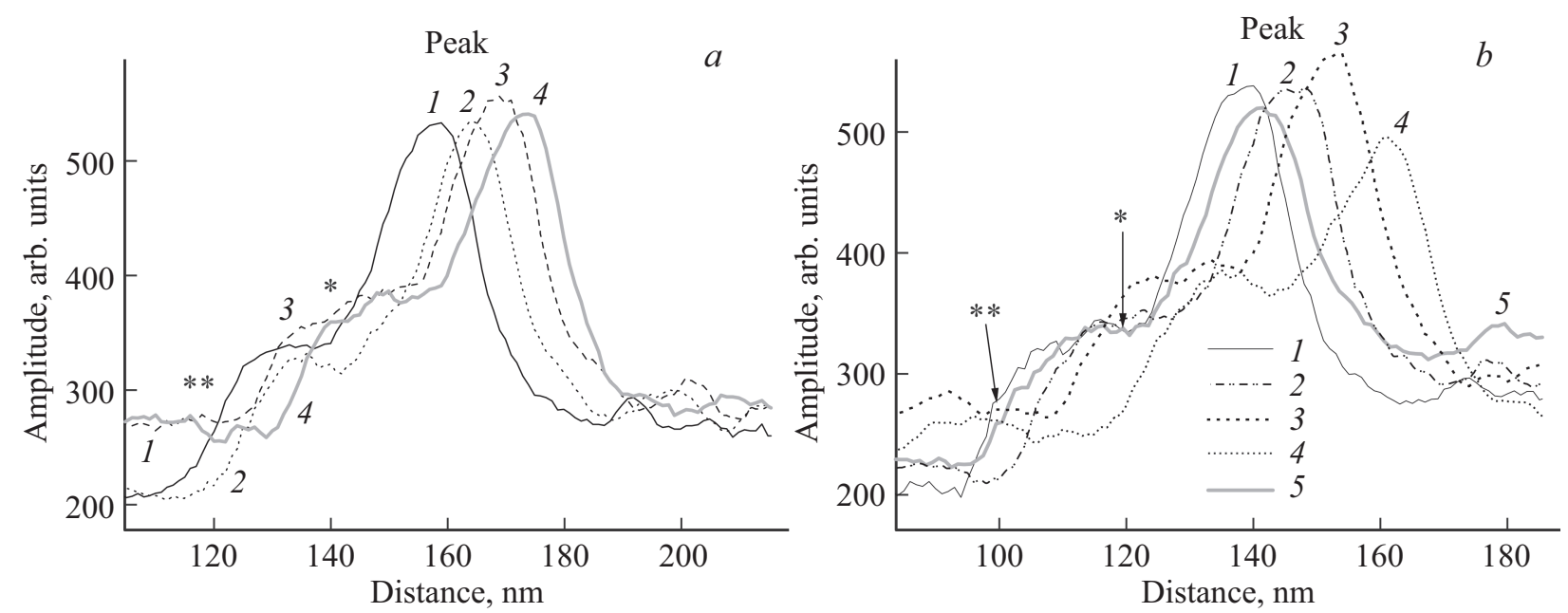

Рис. 3. Участки кривых ВС от левого края выступа $B 1$ после первого (1), 50-го (2), 100-го (3), 150-го (4) сканов второй серии сканирований (кривые $1-4$ смещены вдоль $x$ для удобства их представления $-a$ ). Участки кривых ВС от левого края выступа $B 1$ после пяти серий сканов $(1),(2),(3),(4),(5)$ с промежутком между сериями в несколько месяцев (кривые смещены вдоль $x$ для удобства их представления - $b$ ).

вательных сканов. На рис. 3 показана вариация формы кривой для выступа $B 1$ после нескольких сканов одной серии $(a)$ и после четырех длительных перерывов в последовательности серий сканирования $(b)$.

На рис. 3, a с ростом числа сканирований наиболее заметно изменился уровень ВС от нижнего плато выступа. Эмиссия МВЭ из него возросла более чем на 50 условных единиц после 50-ого скана и затем осталась неизменной до конца серии из 150 сканов. При этом соответствующая вариация эмиссии от верхнего плато этого выступа от начала до конца серии менее заметна (около $\sim 20 \mathrm{arb}$. units и не является монотонной). Это убеждает в том, что наведенная эмиссия зависит от участка сканируемого профиля.

Особенно неоднородными участками являются боковые стенки выступа. Эмиссия от них варьируется сложнее потому, что середина и края стенок эмитируют МВЭ существенно по разному. Участки кривых, которые прилегают к пикам слева, демонстрируют „полочки“ в изменении амплитуды ВС (обозначены знаком *). Полочки формируются эмиссией МВЭ из средней части боковых стенок [4]. Слева и справа от полочек находятся участки ВС, соответствующие фрагментам плато и боковой стенки выступа, которые одновременно облучаются пучком. Фрагменты верхнего плато и боковой стенки формируют пики кривых (РeaK), фрагменты нижнего плато и боковой стенки формируют подъемы, обозначенные знаком **.

Видно (рис. 3,a), что при слабом изменении амплитуды ВС на полочке, ее ширина заметно увеличилась (от $\sim 13$ до $\sim 21 \mathrm{~nm}$ ). Однако, значение ширины выступа, измеренное по точкам, соответствующим границам 
ВО выступа в [4], уменьшалось всего на $\sim 1.7 \mathrm{~nm}$ (разница размера у вершины и основания пика на рис. 2,a), что связано с большей крутизной этого участка кривой ВС. Слабые изменения сигнала ВС произошли на ВО выступа ( 10-20 arb. units).

Вариация формы кривых ВС после перерывов для выступа $B 1$ проявляется поиному. На рис. $3, b$ представлена вариация эмиссии из области левого края выступа $B 1$ в пяти сериях его сканирований, разделенных промежутками времени в несколько месяцев. Рисунок демонстрирует лишь первые кривые ВС в каждой из серий, которые отражают изменения эмиссии, возникшие под воздействием предыдущих сканирований, а также их релаксацию в результате выдержки образца на воздухе.

В этом случае динамика вариации эмиссии на нижнем плато и боковой стенке выступа (и отчасти, в пике ВС) выступа $B 1$ следуют единой закономерности и отличается от соответствующей динамики на верхнем плато. Отметим, что изменения эмиссии, отмеченные вариацией кривых $2-4$ на рис. $3, b$, релаксировали в итоге к кривой 5 , близкой по форме и значению ВС кривой 1. Это свидетельствует о том, что все эти вариации эмиссии связаны с изменением зарядового состояния, а не с контаминацией. Отметим, что вариация зарядового состояния довольно сложна. Если после первой серии сканов наведенная эмиссия на нижнем плато и боковой стенке (кривые 4 и 1 на рис. 3, $a$ ) заметно релаксировала после перерыва в сканировании (кривые 2 и 1 на рис. $3, b$ ), то после последующих серий она только возрастала, уменьшившись повторно лишь после четвертого перерыва до исходного уровня. При этом на верхнем плато динамика вариации эмиссия для кривой 5 отлична от соответствующей на нижнем плато (кривые $1-5$ на рис. $3, b$ ).

Ширина полочек на кривых $1-5$ рис. $3, b$ монотонно уменьшалась (в отличие от ширины на рис. $3, a$ ) с $\sim 20$ до $\sim 17 \mathrm{~nm}$ с правой и левой сторон выступа, в целом на выступ уменьшение составило $\sim 6 \mathrm{~nm}$. При этом, увеличение значений ширины выступов, определенное по рис. 2, $а$ по верхнему основанию, составило $\sim 4.5 \mathrm{~nm}$, а увеличение ширины выступа, определенное по серединам участков подъема (обозначенных **) на кривой ВС на рис. $3, b$, оказалось равным $\sim 1-2 \mathrm{~nm}$. Эти данные подтверждают вывод о сокращении ширины полочек выступа $B 1$ в результате четырех серий сканирования. Кривые 1 и 5 на рис. $3, b$ совмещены таким образом, что бы факт уменьшения ширины полочки оказался наглядным.

Вариация кривых ВС от выступа $B 2$ представлена на рис. 4 для 3 серий сканирования с двумя длительными перерывами. Вариация кривых для этого выступа заметно отличается от соответствующей для выступа $B 1$. Для кривых на рис. 4 можно видеть почти одинаковое увеличение эмиссии для всех участков кривых к концу каждой серии (кривые 2 и 1,4 и 3) и ее релаксацию после перерывов (кривые 2 и 3,4 и 5 и особо заметно 5

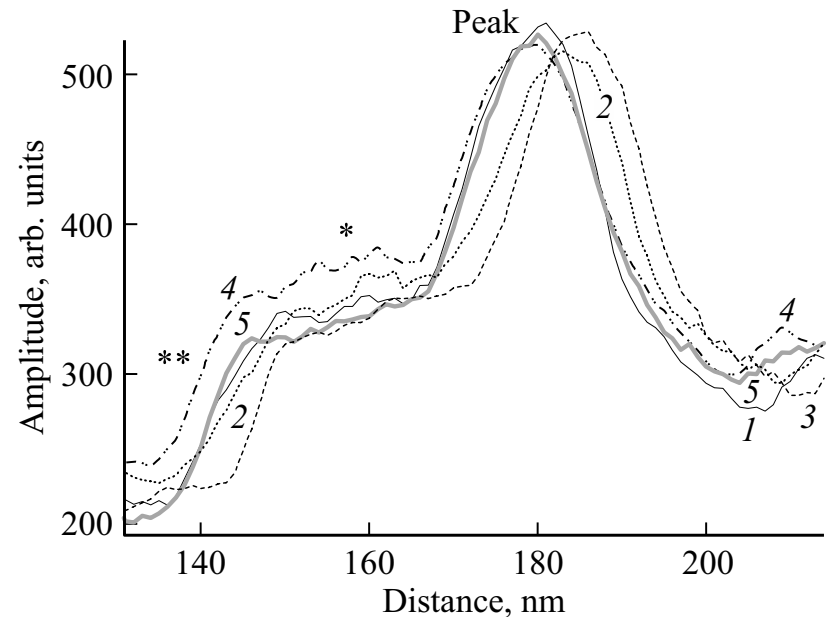

Рис. 4. Участки кривых ВС от левого края выступа $B 2$ после первого (1) и 150-го сканов (2) в первой серии сканирования, а также после первого скана (3) и 150-го сканов (4) в следующей серии и после первого скана в 3 серии (5). РеаК-пики кривой, * - участки-полочки, соответствующие боковой стенке, ${ }^{* *}$ - участки подъема, соответствующие фрагментам нижнего плато и боковой стенки, облучаемым одновременно.

и 4) на 20-30 условных единиц. В отличие от выступа $B 1$ ширина полочек на выступе $B 2$ в конце каждой серии увеличивалась на $\sim 2-3 \mathrm{~nm}$, с релаксацией этого увеличения после перерывов. Это свидетельствует о наведенном увеличении ширины полочек, при этом рис. $2, b$ демонстрирует наведенное уменьшение ширины BO также на $\sim 2 \mathrm{~nm}$.

\section{5. Обсуждение результатов}

Характер вариации эмиссии МВЭ от выступов сильно зависит от выбранного участка их поверхности. Наиболее важными в метрологическом отношении деталями профиля являются участки вблизи верхних и нижних углов выступа. Эмиссия МВЭ от участков вблизи верхних углов изменяется особенно сложным образом. В начале сканирования пики ВС от обоих краев выступа смещаются со слабым изменением своей формы к центру изображения для всех исследованных выступов (в частности, и на рис. $2, a, b)$. Но после перерывов в сканировании пики стремятся восстановить исходное положение и форму. Это означает, что расстояние между пиками кривой не определяется лишь геометрическими краями выступа, как в теории, а зависит также от неизвестной влияющей величины.

После начального сближения всех пиков кривой ВС (и уменьшения ширины верхнего основании выступов) в характере изменения их положения начинается другой этап. Действительно, после первых 10-20 сканов „быстрой“ фазы вариации для разных выступов в ходе второй, „медленной“ фазы зафиксировано либо удаление пиков друг от друга, либо постепенная стабилизация 


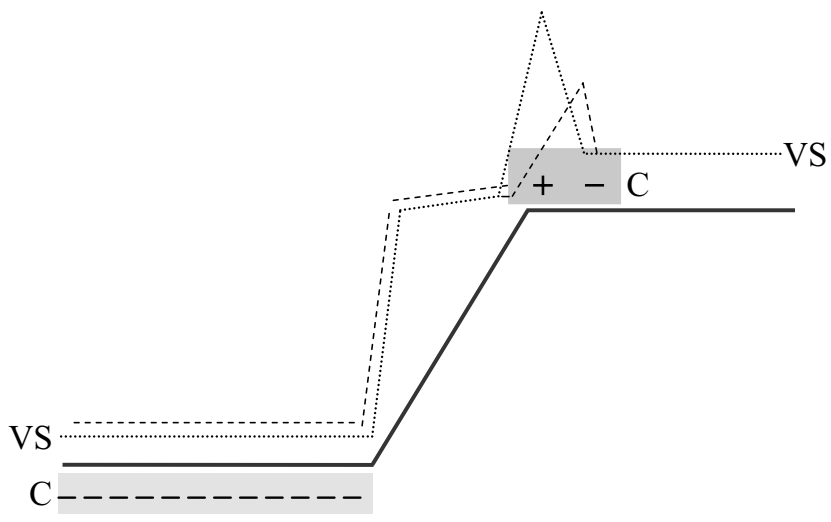

Рис. 5. Схема профиля левой части выступа. Серыми прямоугольниками обозначены участки с наведенными отрицательными $(-)$ и положительными $(+)$ зарядами - С. Точечная линия представляет модель кривой ВС для первых сканов, штриховая - для последних.

расстояния между ними, либо даже его уменьшение. Характер изменения положения пиков в медленной фазе устойчиво воспроизводился для одних выступов и отличался от соответствующего характера для других. Разные фазы изменения положения пиков должны быть обусловлены качественно разными процессами, протекающими на поверхности выступов.

На наш взгляд, определяющая роль в изменении формы кривой ВС в быстрой и медленной фазах принадлежит изменению зарядового состояния участков поверхности на боковой стенке и вблизи ее краев на верхнем и нижнем плато выступа. При этом, в медленных фазах динамика изменения зарядового состояния для выступов $B 1$ и $B 2$ должна быть существенно разная.

В быстрой фазе под поверхностью большей части склона и нижнего плато выступов $B 1$ и $B 2$ предположительно наводятся отрицательные заряды, увеличивающие эмиссию МВЭ из этих участков. При этом, на узких фрагментах профиля вблизи внешней стороны верхних углов выступа локально собираются положительные заряды, в результате чего эмиссия из этих фрагментов уменьшается и на соответствующих внешних участках пика кривых уменьшается амплитуда ВС. При этом с внутренней стороны от верхних углов наводятся отрицательные заряды и эмиссия от соответствующих им внутренних участков слегка увеличивается, в результате чего положение макушки пика сдвигается к центру изображения выступа. Это приводит к уменьшению значения ширины верхнего основании выступов $B 1$ и $B 2$. После перерыва в сканировании с релаксацией наведенных зарядов значения ширины ВО выступа почти восстанавливаются (рис. 2, $a, b$ ). На рис. 5 показана схема наведения зарядов на левой части выступа в конце длительного сканирования выступа.

После быстрой фазы сканирования с общей для выступов $B 1$ и $B 2$ картиной перераспределения зарядов на их поверхности начинаются качественно иные процессы, приводящие к разной для этих выступов вариации кривых в медленной фазе.

На нижнем плато и боковой стенке выступа $B 1$ после второй серии сканирования начинает накапливать нерелаксируемый отрицательный заряд, сохраняющийся во время двух последующих перерывов. Он приводит к увеличению эмиссии из соответствующих фрагментов профиля. Однако, после 4 серии сканирования произошла релаксация и этого заряда. Мы не можем объяснить сложную динамику вариации зарядовых состояний на нижнем плато выступа.

В ходе медленной фазы на верхнем плато выступа $B 1$ происходило, предположительно, высаживание контаминационной пленки. Это увеличивало, как видно из рис. 2, $a$ ширину ВО выступа в ходе каждой серии сканирования. Действительно, ее значение, достигаемое в конце каждой серии сканирований, почти воспроизводилось в начале каждой последующей серии (после участка спада пиков ширины на рис. 2,a). Рост этих значений ширины оказался независимым от роста и релаксации эмиссии, зафиксированной на нижнем плато и боковой стенке выступа $B 1$. Расширение верхнего плато сокращало верхнюю часть боковой стенки, контаминация на которой оказалась слабее. Действительно, уширение выступа по участкам подъема кривой ВС (обозначенных ${ }^{* *}$ ) оказалось вдвое меньше уширения ВО. Уменьшением ширины полочки можно наблюдать визуально при сравнении кривой 5 с кривой 1 на рис. $3, b$.

Эмиссия МВЭ от органической пленки слабее, чем от кремниевой поверхности, покрытой окисной пленкой [13], что должно приводить к потемнению РЭМизображения выступа и к уменьшению контраста рельефной структуры. Однако, пленка, осаждаемая на ВО выступа $B 1$, немонотонно варьировала свою эмиссию в сериях сканирования (рис. $3, b)$. Предположительно, это результат вариации заряда в пленке по мере ее роста.

Таким образом, вариация значения ширины верхнего основания вызывается двумя изменяющимися в ходе сканирования влияющими величинами: осаждением контаминационной пленки, которая покрывает исходные границы верхнего основания, и наводимыми в окисной и контаминационной пленках зарядами. Наведение зарядов на границах верхнего основания приводит к временному смещению пиков кривой ВС, уменьшающему значение ширины в быстрой фазе для выступа $B 1$ и $B 2$. Длительный перерыв в сканировании приводит к релаксации наведенных зарядов.

После стабилизации заряда в пленках в быстрой фазе определяющим фактором изменения ширины выступа $B 1$ в медленной фазе становится, предположительно, селективное осаждение контаминационной пленки на его ВО. Любопытно, что даже для контаминированных краев верхнего плато выступа $B 1$ зафиксирована медленная и быстрая фаза изменения значении ширины (рис. $2, a)$. Это означает, что заряды, ответственные за 
изменение значений ширины, могут наводиться и внутри пленки

Свидетельство смещения пиков под действием наведенных зарядов и возможность существования исходных резидентных зарядов на углах верхнего плато до сканирования выступа в РЭМ ставят вопрос о правомерности предположения о соответствия положения пиков кривой лишь геометрическим границам верхнего основания в существующих моделях эмиссии МВЭ из выступа.

Вариация наведения зарядов на выступе B2 происходила, видимо, почти одинаково на верхнем, нижнем плато выступа и на боковой стенке (кривые 2 и 4 по сравнению с кривыми 1 и 3 на рис. 4). За время прерывания сканирования эмиссия стремилась к восстановлению (кривые 2 и 3 и 4 и 5), а форма кривой 5 после многих серий сканирования почти вернулась к форме кривой 1. Единый характер увеличения и релаксации эмиссии на всех участках выступа $B 2$ свидетельствуют об отсутствии на нем контаминационной пленки. Монотонное уменьшение значений ширины выступа в медленной фазе подтверждает это предположение.

Мы предполагаем, что тип зарядов, наводимых в быстрой фазе на выступах $B 1$ и $B 2$ в слоях окисной и контаминационной пленок, отличаются от типа зарядов, наводимых в этих же слоях в медленной фазе. Последний тип зарядов создает возможность высаживания контаминационной пленки или блокировку этой возможности. И наличие и отсутствие контаминационного уширения означает, что осаждение молекул углеводородов зависит не только от внешних факторов (интенсивности тока в пучке и плотности УВ-молекул на поверхности пластины), но и от пока неизвестной влияющей величины.

\section{6. Заключение}

Обнаружена вариация эмиссии медленных вторичных электронов из рельефной поверхности выступа в ходе длительного ее сканирования в низковольтном РЭМ. Характер вариации сильно отличается для разных участков выступа (верхнее основание, боковые стенки, нижнее плато), но имеет закономерные черты для выбранного участка и воспроизводится на нем при повторных сканированиях.

Наиболее сложная вариация эмиссии возникает от участков профиля вблизи верхних углов выступа, формирующих максимумы эмиссии и пики на кривых РЭМ-видеосигнала (ВС). Характер вариации на них двухэтапный. На первом этапе сканирования выступа обычно происходит смещение максимумов эмиссии к центру выступа, что приводит у сближению пиков кривых ВС и к уменьшению значения ширины выступа. На втором этапе продолжаемого сканирования для разных выступов происходит дальнейшее сближение или расхождение пиков. Перерывы в сканировании приводят к восстановлению исходного положения участков с максимальной эмиссией и к исходному положению пиков кривой. Положение пиков, смещенных на втором этапе, после перерывов не восстанавливалось. Их последующее смещение продолжало тенденцию, сформированную в предыдущих сканированиях.

Вариация эмиссии на первом этапе объяснена генерацией, накоплением и релаксацией локализованных зарядовых состояний в приповерхностном окисном и, возможно, в контаминационном слоях выступа. Под действием электронного пучка в вакууме зарядовые состояния на время смещаются вдоль поверхности, а во время длительных перерывов в сканировании они либо возвращаются на прежнее место, либо сохраняются и даже постепенно накапливаются.

Локализованные зарядовые состояния на разных участках рельефной поверхности имеют разные свойства и влияют на вариацию эмиссии от соответствующих участков с разным эффектом. Эти различия можно объяснить большим разнообразием возможных типов точечных дефектов в слое естественного окисла. Сложное изменение зарядовых состояний на углах выступа можно дополнительно объяснить большей плотностью в их расположении, невоспроизводимой толщиной окисной [14] и контаминационной пленок вблизи угловых участков выступа.

Локализованные зарядовые состояния на поверхности выступа влияют на осаждение частиц углеводорода и определяют, по-видимому, неравномерное осаждение на нее контаминационной пленки. Это приводит к неконформному изменению геометрической формы выступа, к смещению участков максимальной эмиссии и пиков на кривой ВС. Положение смещенных пиков не восстанавливается после прерывания сканирования, что позволяет определить наличие контаминации. На нескольких выступах зафиксировано отсутствие контаминационного уширения выступа. Это объяснено блокированием осаждения пленки также в результате формирования на краях выступа особого зарядового состояния.

Вариация эмиссии в результате сканирования привела к изменению значений геометрических размеров выступов в нанометровом диапазоне. Предельное увеличение значения ширины выступа в ходе длительного сканирования (до 20000 кадров) составило $\sim 4.5 \mathrm{~nm}$, а в другом предельном случае оно уменьшилось на $\sim 0.8 \mathrm{~nm}$.

\section{Конфликт интересов}

Авторы заявляют, что у них нет конфликта интересов.

\section{Список литературы}

[1] Ю.В. Ларионов, Ю.В Озерин. Нано- и микросистемная техника. 11, 650 (2016).

[2] Ю.В. Ларионов. Нано- и микросистемная техника. 19, 323 (2017). 
[3] Э.И. Рау, А.А. Татаринцев, Е.Ю. Зыкова, И.П. Иваненко, С.Ю. Купреенко, К.Ф. Миннебаев, А.А. Хайдаров. ФТТ 59, 599 (2017).

[4] Ю.А. Новиков, Ю.В. Озерин, Ю.И. Плотников, А.В. Раков, П.А. Тодуа. В сб.: Линейные измерения микрометрового и нанометрового диапазонов в микроэлектронике и нанотехнологии / Под ред. Ю.А. Новикова. Тр. ИОФАН. Наука, M. (2006). C. 36.

[5] В.А. Гриценко. УФН. 179, 921 (2017).

[6] М.Н. Левин, А.В. Татаринцев, Е.В. Бондаренко, В.Р. Гитлин, В.А Макаренко, А.Е. Бормонтов. Вестн. ВГУ. Сер. Физика, математика. 2, 30 (2008).

[7] M. Morita, T. Ohmi, E. Hasegava, V. Kawakami, M. Ohwada. J. Appl. Phys. 68, 1272 (1990).

[8] Ю.В. Иванков, М.Н. Левин, В.Р. Гитлин, С.Г. Кадменский. Моделирование радиационных дефектов в структурах металл-диэлектрик-полупроводник. Мин. обр. и науки РФ, ВГУ. (2003). С. 43.

[9] K. Kanaya, S. Okayama. J. Phys. D 5, 43 (1972).

[10] И.М. Бронштейн, Б.С. Фрайман. Вторичная электронная эмиссия. Наука, М. (1969). 407 с.

[11] Программная платформа NDPL. https://yadi.sk/i/YtOL8GEid9nK4. (2009).

[12] Ю.В. Ларионов, В.Б. Митюхляев, М.Н. Филиппов. Поверхность. 9, 54 (2008).

[13] Э.И. Рау, Е.Н. Евстафьева, М.В. Андрианов. ФТТ 50, 4, 53 (2008).

[14] M.N. Fillipov, M.A. Ermakova, V.P. Gavrilenko. Proc. SPIE. 8700, 87000U1-6 (2012).

Редактор Д.В. Жуманов 